\title{
IMAGE SEGMENTATION USING CURVE EVOLUTION AND REGION STABILITY
}

\author{
Baris Sumengen, B. S. Manjunath, and Charles Kenney \\ Department of Electrical and Computer Engineering \\ University of California, Santa Barbara, CA 93106-9560 \\ \{sumengen, manj, kenney\}@ece.ucsb.edu
}

\begin{abstract}
A novel scheme for image segmentation is presented. An image segmentation criterion is proposed that gathers similar pixels together to form regions and creates boundaries between two dissimilar regions. This criterion is formulated as a cost function. This cost function is minimized by using gradient-descent methods, which leads to a curve evolution equation that segments the image. The proposed method generalizes previous methods to more complex similarity and distance measures and can be applied to vector valued images such as texture and color images.
\end{abstract}

\section{INTRODUCTION}

Image segmentation is a basic step in many image processing and computer vision tasks. Previous approaches to image segmentation include filtering-based methods to detect edges followed by edge linking, curve evolution and active contour models $[1,5,6,7,8]$, region growing and merging, global optimization based on energy functions and Bayesian criteria, and graph partitioning and clustering. Some of these methods seek to provide a unified framework that enables segmentation based on multiple heterogeneous attributes such as texture, color, and gray level intensity.

Curve evolution methods have been used for image segmentation for over a decade. Some of these methods utilize the geometric nature of the curves to evolve them, and some of them use a cost function to guide the curve evolution. In this paper we define an image segmentation criterion and formulate it as a cost function. This cost function is then used to guide the curve evolution to segment the image into homogenous and distinct regions.

In comparison to the previous work [5,7], where simple statistics such as intensity mean and standard deviation are utilized, the proposed method lays a generalized framework where the previous methods can be thought as special cases. This flexible framework accepts wide variety of features for similarity of pixels, such as their intensity, texture and color features as well as the distance between the pixels.

The rest of the paper is organized as follows. We review curve evolution methods in section 2. In section 3 , we present a region-based approach to segmentation using geometric active contours. In section 4 we present some experimental results and conclude with discussions in section 5 .

\section{PREVIOUS WORK}

Active contours and curve evolution methods usually define an initial contour $C_{0}$ and deform it towards the object boundary. The problem is usually formulated using partial differential equations (PDE). The previous research follows two different paths in terms of representation and implementation of active contours, namely parametric active contours (PACs) and geometric active contours (GACs). PACs use a parametric representation of the curves and GACs utilize level set methods [2,3]. Level set methods can easily handle topology changes of the evolving contour such as splitting and merging, and singularities on the curve such as sharp corners. Recently some connections between these two methods have been established [1,4]. A summary and comparison of both GACs and PACs can be found in [4].

Curve evolution methods can be classified into several groups: edge-based [1], region-based [5,6,7] and hybrid [8] active contours. Our implementation in this paper is based on region-based GAC methods.

Region-based active contour methods attempt to partition the image into two regions: foreground and background. They start with an initial closed contour and modify the curve according to the statistics of the interior and exterior of this contour. Region-based methods use global image features as opposed to the local features used in edge-based methods.

Developments in region-based active contours [9] are more recent than their edge-based counterparts. Regionbased active contours are less dependent on the initial location of the contour since they don't rely much on the local image features. Also not needing to use the gradient of the image simplifies both the variational formulation and its solution. Region-based methods are also easier to extend to vector valued images such as color and texture images.

Let $C(\varphi):[0,1] \rightarrow \mathfrak{R}^{2}$ be a parameterization of a 2-D closed curve, $I$ be a function defined on a closed region $R, R_{i}$ and $R_{o}$ be the interior and the exterior of $C, m_{i}$ and $m_{o}$ be the corresponding means, $A_{i}$ and $A_{o}$ the areas of $R_{i}$ and $R_{o}$ respectively, $I_{i}$ be $I\left(R_{i}\right)$ defined on $R_{i}$ and $I_{o}$ be $I\left(R_{o}\right)$ defined on $R_{o}$. Tsai, et al. [5] define their optimization criteria as maximizing the separation of the 
mean values: $\left(m_{i}-m_{o}\right)^{2}$. This leads to a gradient-descent equation

$$
\frac{\partial C}{\partial t}=\left(m_{i}-m_{o}\right)\left(\frac{I-m_{i}}{A_{i}}+\frac{I-m_{o}}{A_{o}}\right) \vec{N}-\gamma \kappa \vec{N}
$$

where $\vec{N}$ is the normal vector to $C, \kappa$ is the curvature and $\gamma$ is a constant weighting factor. The $\kappa$ dependent term is added to keep the curve smooth at all times.

Chan, et al. [7] on the other hand uses a limiting version of Mumford-Shah functional [10] as the criteria, where the image is modeled with a piecewise constant functions. The resultant gradient-descent equation is:

$$
\frac{\partial C}{\partial t}=\left(m_{i}-m_{o}\right)\left(I-m_{i}+I-m_{o}\right) \vec{N}-\gamma \kappa \vec{N}
$$

Later on Tsai et al. [6] generalized this equation by solving the general Mumford-Shah problem instead of the limiting case.

\section{PROPOSED IMAGE SEGMENTATION}

In this section, our aim is to define a new flow equation for image segmentation that satisfies the following objectives:

1) The evolving curve segments the image into homogenous regions. Homogenous region means that the pixels in this region that are close to each other have similar properties such as their intensity. This criterion for the segmentation groups similar pixels together into regions.

2) The evolving curve establishes boundaries between regions that are distinct in term of their content, which means, pixels corresponding to neighboring regions are not similar to each other. This criterion aims to increase the dissimilarity across the boundaries.

Let $C_{0}$ be a 2-D closed curve partitioning an image $R$ into two regions: the interior $R_{i}$ and the exterior $R_{o}$ of the curve. Let the image $I$ be a continuous function defined on $R$. Let $w\left(p_{i}, p_{j}\right)$ be a positive, symmetric function, which is a measure for the dissimilarity between points $p_{i}$ and $p_{j}, p_{i}, p_{j} \in R$. Examples for $w\left(p_{i}, p_{j}\right)$ are

$$
\begin{gathered}
w\left(p_{i}, p_{j}\right)=\left|I\left(p_{i}\right)-I\left(p_{j}\right)\right| \\
w\left(p_{i}, p_{j}\right)=\left|I\left(p_{i}\right)-I\left(p_{j}\right)\right| e^{\frac{-\operatorname{dist}\left(p_{i}, p_{j}\right)}{\sigma_{\text {dist }}}}
\end{gathered}
$$

where $\operatorname{dist}\left(p_{i}, p_{j}\right)$ is either $\mathrm{L} 1$ or $\mathrm{L} 2$ distance.

To satisfy the first criterion $C_{0}$ needs to be evolved in a way to maximize intra pixel similarities for both $R_{i}$ and $R_{o}$. To achieve this, a cost function is needed to guide the curve evolution. Assuming that the image only consists of a foreground object and a background, minimizing the energy functional

$$
E_{1}=\int_{R_{i}} \int_{R_{i}} w\left(s_{1}, s_{2}\right) d s_{1} d s_{2}+\iint_{R_{o}} R_{o} w\left(s_{1}, s_{2}\right) d s_{1} d s_{2}
$$

defines the desired first criterion.
On the other hand, to be able to satisfy the second criterion, the curve evolution should also increase the dissimilarity of the pixels of $R_{i}$ and $R_{o}$. This corresponds to minimizing another energy functional

$$
E_{2}=-2 \iint_{R_{i}} \int_{o} w\left(s_{1}, s_{2}\right) d s_{1} d s_{2}
$$

Since it is difficult to find the direct solutions for these energy minimization problems, gradient-descent methods are usually utilized.

Lemma 1: Let $C_{1}$ and $C_{2}$ be arbitrary closed curves defined on $R, w: \mathbb{R}^{2} \times \mathbb{R}^{2} \rightarrow \mathbb{R}$ be a real valued function,

$$
E=\int_{R\left(C_{1}\right)} \int_{R\left(C_{2}\right)} w\left(s_{1}, s_{2}\right) d s_{1} d s_{2}
$$

be the cost functional to be minimized by evolving $C_{1}$ and $C_{2}$. The notation $R(C)$ corresponds to the area inside the curve $C$. The first variation and the corresponding gradient descent of this energy functional are as following.

$$
\begin{aligned}
& \frac{\partial E}{\partial t}=\int_{C_{1}}\left\langle\frac{\partial C_{1}}{\partial t},\left(\int_{R\left(C_{2}\right)} w\left(c_{1}, s\right) d s\right) \vec{N}_{1}\right\rangle+\int_{C_{2}}\left\langle\frac{\partial C_{2}}{\partial t},\left(\int_{R\left(C_{1}\right)} w\left(c_{2}, s\right) d s\right) \vec{N}_{2}\right\rangle \\
& \Rightarrow \frac{\partial C_{1}}{\partial t}=-\int_{R\left(C_{2}\right)} w\left(c_{1}, s\right) d s \vec{N}_{1}, \frac{\partial C_{2}}{\partial t}=-\int_{R\left(C_{1}\right)} w\left(c_{2}, s\right) d s \vec{N}_{2}
\end{aligned}
$$

where $\vec{N}_{1}$ and $\vec{N}_{2}$ are the outwards normal vectors for $C_{1}$ and $C_{2}$ respectively, $c_{1} \in C_{1}$ and $c_{2} \in C_{2}$.

Proof: A full proof is out of the scope of this paper because of space restrictions. Intuitively the results can be achieved as following. From previous work [11], we know that the first variation and the gradient-descent for the functional $K=\int_{R(C)} H(s) d s$ is

$$
\frac{\partial K}{\partial t}=\int\left\langle C_{t}, H \vec{N}\right\rangle \quad \Rightarrow \quad \frac{\partial C}{\partial t}=-H \vec{N}
$$

By taking $H=\int_{R(C)} w(c, s) d s$, where $C=C_{2}$ and $C=C_{1}$ the results in Lemma 1 follow. $\square$

Corollary 1: If $C=C_{1}=C_{2}$ in (7), which means $\vec{N}=\vec{N}_{1}=\vec{N}_{2}$, then

$$
\frac{\partial C}{\partial t}=-2 \int_{R_{i}} w(c, s) d s \vec{N}
$$

Corollary 2: If $C=C_{1}=-C_{2}$ in (7), which means $\vec{N}=\vec{N}_{1}=-\vec{N}_{2}$, then

$$
\frac{\partial C}{\partial t}=\left(\int_{R_{o}} w(c, s) d s-\int_{R_{i}} w(c, s) d s\right) \vec{N}
$$

Using corollaries 1 and 2, we notice that both (5) and (6) result the same gradient descent equation. This is not surprising considering the relation 


$$
E_{1}=E_{2}+\underbrace{\int_{R R} w\left(s_{1}, s_{2}\right) d s_{1} d s_{2}}_{\text {A Constant }}=E_{2}+k
$$

where $k$ is a constant. The gradient-descent flow corresponding to both $E_{1}$ and $E_{2}$ is

$$
\frac{\partial C}{\partial t}=2\left(\int_{R_{o}} w(c, s) d s-\int_{R_{i}} w(c, s) d s\right) \vec{N}
$$

where $c$ is a point on the curve $C$. Since (10) is a solution for both $E_{1}$ and $E_{2}$, this curve evolution satisfies both of our objectives of having consistent regions with high intra similarity and having dissimilar neighboring regions.

To demonstrate how (10) works, consider the binary image in Fig. 1. Let this image consist of a foreground object $I_{i}$ and the background $I_{o} . I_{i} \cup I_{o}=R$. The curve $C$ is initialized inside $I_{i}$ leading to $R_{i} \subset I_{i}$. Applying (10) to the points on the curve $\mathrm{C}$, the integral over $R_{i}$ is equal to zero, whereas the integral over $R_{o}$ results a positive number expanding the curve towards the object boundaries. If the curve passes the object boundary and reaches the background $I_{o}$, (10) becomes negative while shrinking the curve back.

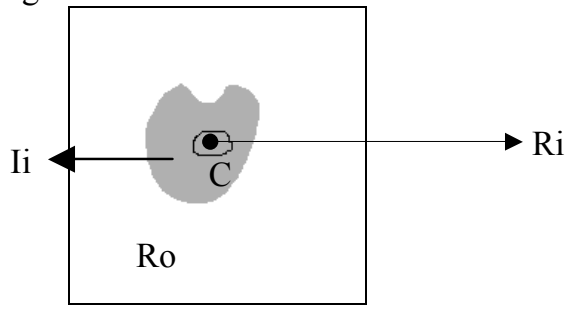

Figure 1. Binary image: A small curve inside the foreground object evolves to capture the object boundaries.

For demonstration purposes, we simplified several things in our formulation of (5) without loss of generality. Equal weightings are assumed for both areas $R_{i}$ and $R_{o}$ and in addition, one commonly used criterion, minimizing the curve length, is also ignored. Including these extra conditions, the solution becomes:

$$
\frac{\partial C}{\partial t}=\left(\alpha \int_{R_{o}} w(c, s) d s-\beta \int_{R_{i}} w(c, s) d s\right) \vec{N}-\gamma \kappa \vec{N}
$$

where $\kappa$ is the curvature, which smoothes out the boundaries. We choose $\alpha=1 / A_{o}$ and $\beta=1 / A_{i}$ in our implementation if (3) is used as the dissimilarity measure. Even though the areas $A_{i}$ and $A_{o}$ are not constants but functions of time, it can be shown that this evolution equation still decreases the proposed energy functional, so it is a descent equation. Moreover using the areas as weighting factors has a geometric meaning of normalization. Geometric criteria have been widely utilized in the past for curve evolution $[1,2,3]$ and laid the foundations of GACs.

To apply the proposed curve evolution to vectorvalued images, such as color and texture images, there are two ways of doing it. The first approach, which is the traditional way, is to create $\mathrm{N}$ coupled curve evolutions, each applied to one of the dimensions of the $\mathrm{N}-\mathrm{d}$ vector valued image. In this method, the complexity increases linearly with the number of dimensions. The second approach is to calculate $w(x, y)$ using vector values and not change the PDE. For example, the extended version of (3) is

$$
w(x, y)=\sum_{N}\left|V_{i}(x)-V_{i}(y)\right|
$$

Since the PDE stays unchanged, the computational complexity also stays the same.

Expanding the segmentation to multiple regions is also possible by recursively applying the bi partitioning to each region.

\section{EXPERIMENTAL RESULTS}

Fig. 2 shows the flow of the segmentation algorithm. Starting with the input image I, the dissimilarity matrix W and its elements $w\left(s_{i}, s_{j}\right)$ are calculated using (3) or (4). An initial curve $C_{0}$ is propagated under the $\kappa$ and $\mathrm{W}$ based forces until it converges.

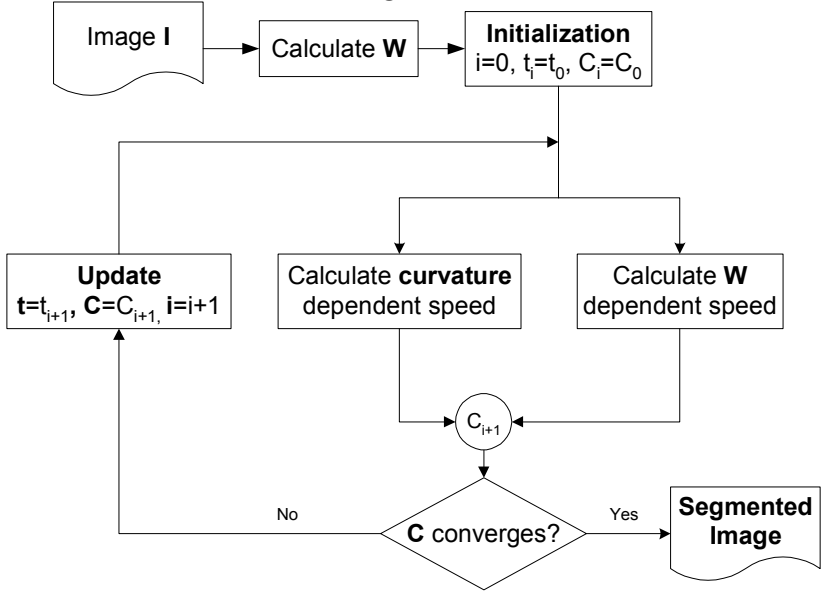

Figure 2. Flow diagram of the segmentation algorithm

The curve can be instantiated manually or automatically placing curves at random or organized places on the image. As demonstrated in Fig. 3, the proposed segmentation method does not depend on the initial location of the curve as much as the edge-based active contours [1]. This is because of the use of all image pixels for the curve movement.

We use the well-known level set method formulation $[2,3]$ to implement the curve evolution in (11). This requires defining a corresponding level set function $U$ that embeds $C$ as its zero level set and the time evolution of $U$. The level set equation corresponding to (11) is

$\frac{\partial U}{\partial t}=\left(\alpha \int_{R_{o}} w(u, s) d s-\beta \int_{R_{i}} w(u, s) d s-\gamma \nabla \cdot \frac{\nabla U}{|\nabla U|}\right)|\nabla U|$

Here $U$ is a 3-D function where $U(x, y)=0$ defines the evolving curve, and $u$ is a point on $R$. $U$ is generated from the initial curve using the signed distance function 
$U(x, y, t=0)= \pm d$ where $d$ is the distance from $(x, y)$ to $C$ and the sign is chosen positive if $(x, y)$ is outside the contour $C$ and negative if inside the contour.

We have tested the segmentation method on different data sets. The images are intentionally darkened to clarify the evolution of the curve. Segmentation result on an image of blood cells is shown in Fig. 4. $w$ are calculated using intensity values using (3) and an initial curve is evolved to segment the image.

Fig. 3 shows segmentation of a texture image consisting of two regions. A triangle shaped curve is instantiated overlapping both texture regions. $w$ are calculated using (12). The curve evolves and finds the correct boundary.

\section{COMPARISON TO OTHER REGION-BASED GACs}

Previously used energy functionals focused on approximating the regions with a constant or a smooth function. The curve evolution from Chan et al. [7] uses a piecewise-constant model for the images, where their energy functional serves a similar purpose as the first segmentation criterion we proposed. On the other hand, it doesn't consider the dissimilarity of $R_{i}$ and $R_{o}$. Tsai et al.

[5] use $\left(m_{i}-m_{o}\right)^{2}$ as the criteria to maximize the separation of the means $m_{i}$ and $m_{o}$ of $R_{i}$ and $R_{o}$. This objective is similar to our second segmentation criterion. But this method doesn't consider the homogeneity of the regions itself.

\section{REFERENCES}

[1] V. Caselles, R. Kimmel, and G. Sapiro, "Geodesic Active Contours," International Journal of Computer Vision, Feb.March 1997.

[2] S. Osher, J. A. Sethian, "Fronts propagating with Curvature dependent Speed: Algorithms Based on Hamilton-Jacobi Formulations," Journal of Computational Physics, 79, 1988.

[3] J. A. Sethian, "Level set methods and fast marching methods: evolving interfaces in computational geometry, fluid mechanics, computer vision, and materials science", Cambridge University Press, 1999.

[4] C. Xu, A. Yezzi, L. Prince, "On the Relationship between Parametric and Geometric Active Contours", Technical Report JHU/ECE 99-14, Dec 1999.

[5] A. Tsai, A. Yezzi, A.S. Willsky, "A statistical approach to snakes for bimodal and trimodal imagery”, ICCV 1999.

[6] A. Tsai, A. Yezzi, A.S. Willsky, "A curve evolution approach to smoothing and segmentation using the MumfordShah functional", CVPR 2000.

[7] T. F. Chan, L. A. Vese, "Active contours without edges", IEEE Trans. on Image Processing, Feb. 2001.

[8] N. Paragios, R. Deriche, "Geodesic active regions for supervised texture segmentation”, ICCV 1999.

[9] S. C. Zhu, A. L. Yuille, "Region competition: unifying snakes, region growing, and Bayes/MDL for multiband image segmentation”, PAMI 1996.

[10] D. Mumford, J. Shah, "Boundary detection by minimizing functionals", CVPR 1985.

[11] Andy Tsai, Appendix A, PhD Thesis, 2000.

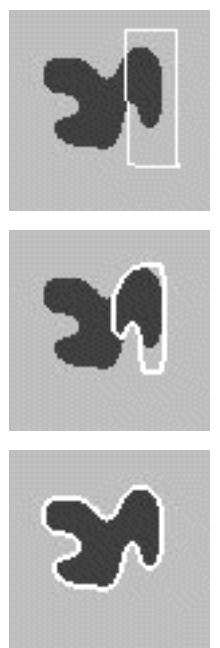

(a)
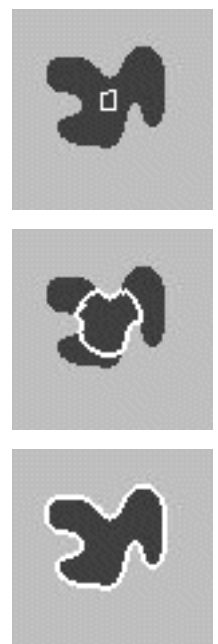

(b)
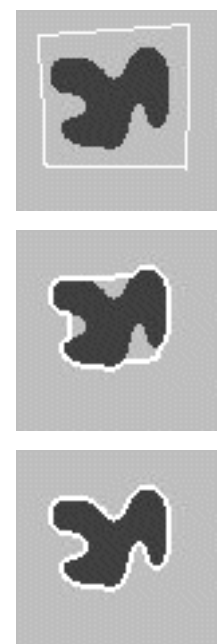

(c)
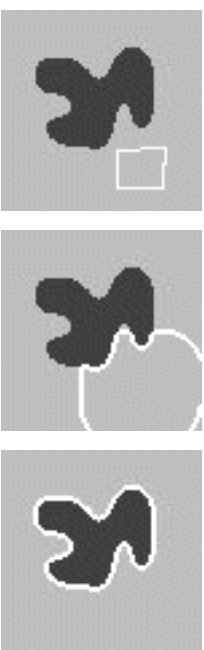

(d)
Figure 3. (a-d) Four different types of locations to instantiate the curve and the corresponding curve evolutions converging to the same solution.

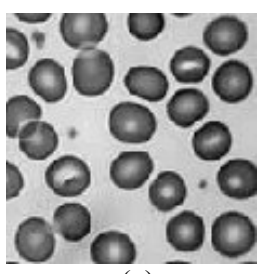

(a)

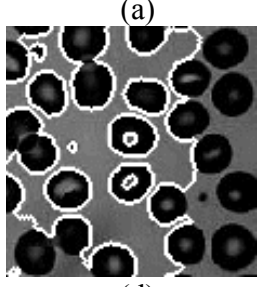

(d)

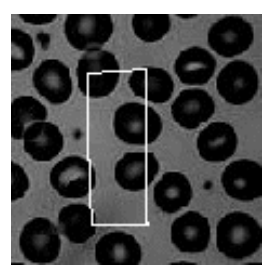

(b)

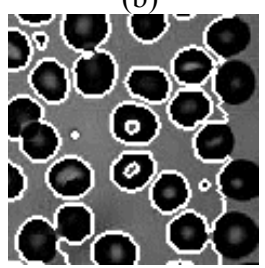

(e)

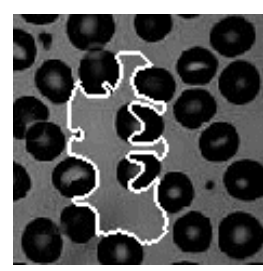

(c)

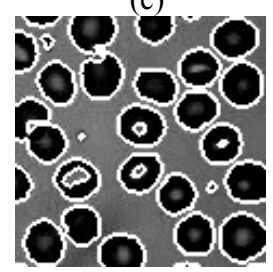

(f)
Figure 4. (a) Original image (b) Initial curve (c-f) Curve evolution using intensity.

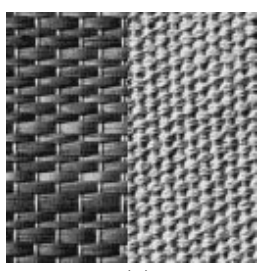

(a)

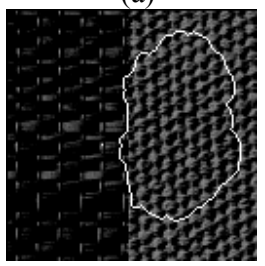

(d)

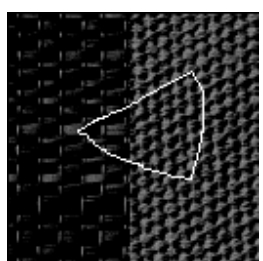

(b)

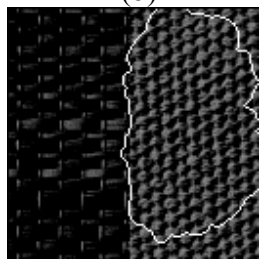

(e)

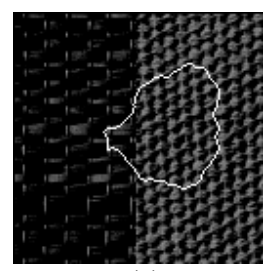

(c)

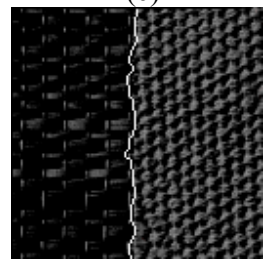

(f)
Figure 5. (a) Original texture image (b) Initial curve (c-f) Curve evolution using texture features. 